\title{
Influence of Electric Acupuncture on Bcl-xl and Caspase-3 Expression After Cerebral Ischemia of Rats
}

\author{
Kangbai Huang, Liang Zhang, Guoxin Zhong \\ Guangzhou University of Chinese Medicine, Guangzhou Guangdong, 510006, China
}

\begin{abstract}
Keywords: Cerebral ischemia, Electric acupuncture, Bcl-xl, Caspase-3.
\end{abstract}
\begin{abstract}
Objective: to study the influence of electric acupuncture on factors about cerebral nerve cell apoptosis: B-cell lymphocytic lymphoma-xl gene (Bcl-xl) and aspartic acid proteolytic enzyme-3 including cysteine (Caspase-3) after cerebral ischemia of rats, and discuss the possible mechanism of treating cerebral ischemia and suppressing cell apoptosis with electric acupuncture. Method: 250 healthy and adult male SD rats were chosen as the objects of study, and they were randomly divided into sham-operation group, model group, electric acupuncture group, AG490 group, and AG490 electric acupuncture group. Each group was divided into 5 sub-groups according to time bucket. There were 25 groups in total. Each group included 10 rats. AG490 right ventricle injection was conducted for AG490 group and AG490 electric acupuncture group 20min before the operation. Baihui point and Dazhui point were chosen for electric acupuncture group and AG490 electric acupuncture for electric acupuncture treatment. The number of immune positive cells of cerebral ischemia nidi of rats Bcl-xl and Caspase-3 was observed. Results: sham-operation group has no significant change in each time bucket after the operation. After cerebral ischemia for $2 \mathrm{~h}, \mathrm{Bcl}-\mathrm{xl}$ and Caspase-3 expression of rats in each group rises, higher than the rats in the sham-operation group in the same time bucket. For Bcl-xl expression, the model group and the electric acupuncture group are most significant, while the expression of AG490+electric acupuncture group is lower than that of electric acupuncture group $(\mathrm{P}<0.01)$. Caspase- 3 content has no difference for each intervention group. $\mathrm{Bcl}$-xl expression of rats in each group after ischemia for $1 \mathrm{~d}$ is basically same with the situation of rats in each group after ischemia for $2 \mathrm{~d}$. The electric acupuncture group presents continuous rising trend. Caspase-3 expression of each intervention group continues to rise. The model group and AG490 group are in the high level $(\mathrm{P}<0.01)$. AG490+electric acupuncture group is lower than the model group $(\mathrm{P}<0.05)$, but AG490+electric acupuncture group has no difference with AG490 group $(\mathrm{P}>0.05)$. The electric acupuncture group is lower than the above three groups $(\mathrm{P}<0.05)$. After cerebral ischemia for $3 \mathrm{~d}, \mathrm{Bcl}-\mathrm{xl}$ expression of rats in each group starts to decline. High-level expression of electric acupuncture group can maintain for a long time, and the content is higher than that of model group $(\mathrm{P}<0.05)$. The content of AG490 group and AG490+electric acupuncture group is low $(\mathrm{P}<0.01)$. The content of Caspase-3 reaches the peak after cerebral ischemia for $3 \mathrm{~d}$. The content of AG490 group is highest $(\mathrm{P}<0.05)$, and the content of electric acupuncture group is lowest $(\mathrm{P}<0.01)$. The model group and AG490+electric acupuncture group are between the two, and the difference of the two is not significant ( $\mathrm{P}>0.05$ ). After cerebral ischemia for $7 \mathrm{~d}-21 \mathrm{~d}$, Bcl-xl and Caspase-3 content of rats in each group continues to drop. After 21d, the differences of rats in each group are not significant $(\mathrm{P}>0.05)$. Conclusion: electric acupuncture can effectively suppress cerebral nerve cell apoptosis after cerebral ischemia, enhance Bcl-xl expression in brain tissue and down regulate Caspase-3 content. It may protect the brain through activating JAK2/STAT5 signal channel.
\end{abstract}

\section{Introduction}

Usually, when ischemia happens to brain tissue, selective necrosis will appear to nerve cells in cerebral ischemia zone and other vulnerable zones, which is also an important cause for cripple and death after cerebral ischemia. Thus. Suppression of cerebral nerve cell apoptosis is the key to treatment of cerebral ischemia ${ }^{[1]}$. Electric acupuncture is a common stimulation therapy. To explore the influence of electric acupuncture on cerebral nerve cell apoptosis of rats after cerebral ischemia, 
250 healthy and adult male SD rats were chosen for grouping and study. Middle cerebral artery occlusion model was established to observe and compare the number of $\mathrm{Bcl}$-xl and Caspase-3 immune positive cells of rats under various interventions. The report is made as follows.

\section{Materials and methods}

\section{Materials}

250 healthy and adult male SD rats were chosen as the objects of study, and they were randomly divided into sham-operation group, model group, electric acupuncture group, AG490 group, and AG490 electric acupuncture group. Each group was divided into 5 sub-groups according to time bucket (2h, 1d, 3d, 7d and 21d after the operation). There were 25 groups in total. Each group included 10 rats. The weight of rats was between $180 \mathrm{~g}$ and $220 \mathrm{~g}$. All of them were fed with water. The feed was common particle-type rat feed including $23 \%$ protein, $4.7 \%$ fat and $0.24 \%$ sodium salt. The temperature in the room was kept within $18 \sim 26^{\circ} \mathrm{C}$, and the humidity was kept within $50 \% \sim 60 \%$. The dark period was $12 \mathrm{~h}$, and automatic ventilation was supplied for 10-20 times per hour.

\section{Reagents and instruments}

G490 (German Merck Company); dimethyl sulfoxide (DMSO, American Sigma Company); $\mathrm{Bcl}-\mathrm{xl}$ and Caspase-3 rabbit polyclonal antibody (American Abnova Company); goat anti-rabbit IgG labeled with horse radish peroxidase (American Benthyl Company); goat anti-rabbit IgG labeled with fluorescein (Goat Anti-Rabbit IgG/FITC) (BEIJING BIOSYNTHESIS BIOTECHNOLOGY CO., LTD.); protein extraction kit and chemiluminiscence coloration (ECL) kit (BEIJING PPLYGEN TECHNOLOGY CO., LTD.); diaminobenzidine (DAB) color developing agent kit (Wuhan Boster Biological Technology Co., Ltd.)

\section{Methods}

\section{Modeling method}

Except sham-operation, electrocoagulation gun was adopted to establish focal cerebral ischemia model in the middle artery of rats in the other 4 groups $^{[2]} .0 .33 \mathrm{ml} 10 \%$ chloral hydrate solution was taken for intraperitoneal injection of rats. The rats were fixed at upper right lateral position. The skin of rats was cut along the middle point of ear-eye line of rats, and temporalis was separated to make bone window and make middle cerebral artery appear. Electrocoagulation closed-gun was adopted to carry out coagulation treatment of middle cerebral artery so as to form focal cerebral ischemia model. $100000 \mathrm{U}$ penicillin was used for conventional feeding for 1-3d after the operation.

\section{Intervention method}

AG490 right ventricle injection [2]

AG490 right ventricle injection was conducted for the rats in AG490 group and AG490 electric acupuncture group 20min before the operation. 5mg AG490 reagent was dissolved in $1 \mathrm{ml}$ DMSO and $19 \mathrm{ml}$ hydrogen peroxide. Then, intraperitoneal injection of chloral hydrate solution was conducted. The hairs of rats on the heads were shaved off, and the rats were disinfected in an conventional way. The skin was cut; blunt dissection was done for the fascia; the surface of skull was eliminated. Blue ink was used to mark. The hole was drilled at $0.8 \mathrm{~mm}$ behind the marked point till the skull. After surgical field was dry, AG490 solution was solely injected at $.8 \mathrm{~mm}$ behind the marked point. The needle was retained for $3 \mathrm{~min}$. Then, the skin was sutured.

Electric acupuncture treatment

Baihui point and Dazhui point were chosen for electric acupuncture group and AG490 electric acupuncture. 1cun acupuncture needle penetrated into Baihui point, with the depth of 0.5cun. The needle penetrated into Dazhui point vertically, with the depth of 0.3cun. G6850 electric acupuncture apparatus was connected for treatment with the frequency of dilatational wave alternation for 20 times per minute, and once per day. 30min lasted once. The initial treatment started $1 \mathrm{~h}$ after modeling.

\section{Sample collection and detection}

The heads of rats were eliminated and the brain of rats was taken. Coronary slice processing was done for the forebrain. $10 \%$ formalin solution was used for fixation. The coronary slice was 
dehydrated conventionally, and paraffin embedding treatment was conducted. The thickness of slice was $6 \mu \mathrm{m}$. immumohistochemical staining S-P method was used to observe the number of Bcl-xl and Caspase-3 immune positive cells of single brain slice in cerebral ischemia focus of rats in strict accordance with requirements of the kit.

\section{Statistical method}

The experimental result was expressed with mean \pm standard deviation $\left({ }^{\bar{x}} \pm \mathrm{S}\right)$. SPSS18.0 statistical package was applied for data processing. One-way AVOVA was used for inter-group comparison. LSD inspection method was used for inter-group pairwise comparison. $\mathrm{P}<0.05$ means the difference has statistical significance.

\section{Results}

\section{Bcl-xl detection result of ischemia focus cortex of rats in each group}

Table 1. Comparison of Bcl-xl content of focus cortex in different time buckets $\left(\bar{X}_{ \pm \mathrm{S}}\right)$

\begin{tabular}{|c|c|c|c|c|c|c|}
\hline Group & $\mathrm{N}$ & $2 \mathrm{~h}$ & $1 d$ & 3d & $7 \mathrm{~d}$ & 21d \\
\hline Sham-operation group & 10 & $8.12 \pm 1.59$ & $8.58 \pm 1.37$ & $7.98 \pm 1.87$ & $\begin{array}{c}8.46 士 \\
2.08\end{array}$ & $\begin{array}{c}8.27 士 \\
1.84\end{array}$ \\
\hline Model group & 10 & $38.54 \pm 3.04 *$ & $\begin{array}{c}40.08 \pm 3.82 \\
*\end{array}$ & $\begin{array}{c}22.72 \pm 3.13 \\
*\end{array}$ & $\begin{array}{l}16.77 士 \\
2.87 \star\end{array}$ & $\begin{array}{c}7.15 \text { 士 } \\
1.76\end{array}$ \\
\hline $\begin{array}{c}\text { Electric acupuncture } \\
\text { group }\end{array}$ & 10 & $39.88 \pm 2.86 *$ & $\begin{array}{c}43.53 \pm 2.74 \\
* \\
\end{array}$ & $\begin{array}{c}33.64 \pm 3.08 \\
* ※ ※\end{array}$ & $\begin{array}{c}23.49 士 \\
3.64 * ※\end{array}$ & $\begin{array}{c}8.38 \text { 士 } \\
1.63\end{array}$ \\
\hline AG490 group & 10 & $\begin{array}{c}27.52 \pm 3.71 * ※ \\
※ \star \star\end{array}$ & $\begin{array}{l}25.39 \pm 2.94 \\
\star ※ ※ \star \star\end{array}$ & $\begin{array}{c}15.78 \pm 3.53 \\
* ※ \star \star\end{array}$ & $\begin{array}{l}13.93 \pm \\
3.76 * \star\end{array}$ & $\begin{array}{c}7.83 \text { 士 } \\
1.59\end{array}$ \\
\hline $\begin{array}{c}\text { AG490 electric } \\
\text { acupuncture group }\end{array}$ & 10 & $\begin{array}{c}28.23 \pm 3.86 * ※ \\
※ \star \star\end{array}$ & $\begin{array}{l}29.58 \pm 0.02 \\
\star ※ ※ \star \star\end{array}$ & $\begin{array}{c}17.84 \pm 3.14 \\
* ※ \star \star\end{array}$ & $\begin{array}{l}15.16 \pm \\
3.38 * \star\end{array}$ & $\begin{array}{c}8.12 \text { 士 } \\
2.03\end{array}$ \\
\hline
\end{tabular}

Note: comparison with sham-operation group in the same time bucket: $* \mathrm{P}<0.01$; comparison with model group in the same time bucket: $※ \mathrm{P}<0.05, ※ \circledast \mathrm{P}<0.01$; Comparison with electric acupuncture group in the same time bucket: $\star \mathrm{P}$ $<0.05, \star \star \star \mathrm{P}<0.01$;

Table 1 shows that only little Bcl-xl is expressed in the sham-operation group in each time bucket. $\mathrm{Bcl}-\mathrm{xl}$ content of each group rises after cerebral ischemia for $2 \mathrm{~h}$. The rising degree of model group and electric acupuncture group is most significant $(\mathrm{P}<0.01)$. Although the content of AG490 group and AG490+electric acupuncture group is higher than that of sham-operation group $(\mathrm{P}<0.01)$, it is lower than that of model group and electric acupuncture group $(\mathrm{P}<0.01)$. Inter-group differences between model group and electric acupuncture group as well as between AG490 group and AG490+electric acupuncture group are not significant $(\mathrm{P}>0.05)$. Bcl-xl expression of rats in each group after ischemia for $1 \mathrm{~d}$ is basically same with the situation of rats in each group after ischemia for $2 \mathrm{~d}$. Although the differences between model group and electric acupuncture group are still not significant, the content of electric acupuncture group presents a continuous rising trend, and the data are more superior. Bcl-xl level of each group starts to decline after cerebral ischemia for 3d, where the content of electric acupuncture group is still at a high level and higher than that of other groups $(\mathrm{P}<0.01)$, followed by the model group. The expression level of AG490 group and AG490+electric acupuncture group is lower than that of the above two groups $(\mathrm{P}<0.01)$. Bcl-xl expression of each group continues to drop after cerebral ischemia for $7 \mathrm{~d}$, but is still higher than that of sham-operation group in the same time bucket $(\mathrm{P}<0.01)$. Electric acupuncture group shows the highest expression $(\mathrm{P}<0.05)$. the differences of model group, AG490 group and AG490+electric acupuncture group are not significant $(\mathrm{P}>0.05)$. Bcl-xl expression of each group continues to drop after cerebral ischemia for 21d, and the differences with sham-operation group are not significant $(\mathrm{P}>0.05)$.

Caspase-3detection result of ischemia focus cortex of rats in each group

Table 2 shows sham-operation group only has few Caspase- 3 positive staining reaction points in each time bucket. The numerical values of each group start to rise after cerebral ischemia for $2 \mathrm{~h}$, 
higher than the numerical values of sham-operation group in the same time bucket $(\mathrm{P}<0.01)$. The differences of each group are not significant $(\mathrm{P}>0.05)$. The numerical values of each group continue to increase after cerebral ischemia for 1d. The model group and AG490 group are at a high level $(\mathrm{P}<0.01)$. The difference between the two groups are not significant $(\mathrm{P}>0.05)$. Ag490+electric acupuncture group is lower than the model group $(\mathrm{P}<0.05)$, but it has no difference with Ag490 group $(\mathrm{P}>0.05)$. The electric acupuncture group is lower than the above three groups $(\mathrm{P}<0.05)$. The content of Caspase-3 positive reactant reaches the peak after cerebral ischemia for 3d. The content of AG490 group is highest $(\mathrm{P}<0.05)$, and the content of electric acupuncture group is lowest $(\mathrm{P}<0.01)$. The model group and Ag490+electric acupuncture group are between the two, and the differences between the two are not significant $(\mathrm{P}>0.05)$. Caspase-3 positive reactant can still be detected after cerebral ischemia for $7 \mathrm{~d}$. The electric acupuncture group is lower than model group, AG490 group and Ag490+electric acupuncture group $(\mathrm{P}<0.01)$. The differences between model group and AG490 group as well as between AG490 group and Ag490+electric acupuncture group are not significant ( $\mathrm{P}>0.05)$. Ag490+electric acupuncture group is slightly lower than the model group $(\mathrm{P}<0.05)$. Each group continues to decline after cerebral ischemia for $21 \mathrm{~d}$, and the differences with sham-operation group are not significant $(\mathrm{P}>0.05)$.

Table 2. Comparison of Caspase-3content of focus cortex in different time buckets $\left(\bar{X}_{ \pm S}\right)$

\begin{tabular}{|c|c|c|c|c|c|c|}
\hline Group & $\mathrm{N}$ & $2 \mathrm{~h}$ & $1 \mathrm{~d}$ & $3 d$ & $7 d$ & 21d \\
\hline Sham-operation group & 10 & $5.18 \pm 0.52$ & $5.04 \pm 0.43$ & $5.36 \pm 0.47$ & $5.22 \pm 0.34$ & $\begin{array}{c}5.89 士 \\
0.31\end{array}$ \\
\hline Model group & 10 & 23.76士3.14* & $\begin{array}{c}47.29 \pm 3.05 \\
*\end{array}$ & $58.27 \pm 3.26 *$ & $\begin{array}{l}32.43 \pm \\
2.83 *\end{array}$ & $\begin{array}{c}7.13 \text { 士 } \\
0.52\end{array}$ \\
\hline $\begin{array}{c}\text { Electric acupuncture } \\
\text { group }\end{array}$ & 10 & $19.98 \pm 2.93 *$ & $\begin{array}{c}32.12 \pm 2.74 \\
* ※ ※\end{array}$ & $\begin{array}{c}39.21 \pm 3.62 * \\
※ ※\end{array}$ & $\begin{array}{c}21.81 \pm 2.36 \\
* ※ ※\end{array}$ & $\begin{array}{c}5.82 \text { 士 } \\
0.43\end{array}$ \\
\hline AG490 group & 10 & $25.08 \pm 2.82 *$ & $\begin{array}{c}44.86 \pm 2.83 \\
\star \star \star\end{array}$ & $\begin{array}{c}67.88 \pm 4.07 * \\
※ \star \star\end{array}$ & $\begin{array}{c}27.29 \pm 3.14 \\
* \star\end{array}$ & $\begin{array}{c}7.96 \text { 士 } \\
0.54\end{array}$ \\
\hline $\begin{array}{c}\text { AG490 electric } \\
\text { acupuncture group }\end{array}$ & 10 & 21.44 士 $3.02 *$ & $\begin{array}{c}40.95 \pm 3.15 \\
\star ※ \star\end{array}$ & $\begin{array}{c}59.14 \pm 3.58 * \\
\star \star \triangle\end{array}$ & $\begin{array}{c}26.86 \pm 2.92 \\
* ※ \star\end{array}$ & $\begin{array}{c}6.51 士 \\
0.37\end{array}$ \\
\hline
\end{tabular}

Note: comparison with sham-operation group in the same time bucket: $* \mathrm{P}<0.01$; comparison with model group in the same time bucket: $※ \mathrm{P}<0.05, ※ ※ \mathrm{P}<0.01$; Comparison with electric acupuncture group in the same time bucket: $\star \mathrm{P}$ $<0.05, \star \star \mathrm{P}<0.01$; comparison with AG490 group in the same time bucket: $\Delta \mathrm{P}<0.05$;

\section{Discussion}

Traditional Chinese medicine holds that, pathogeneses of cerebral ischemia mainly include yin-yang disharmony of viscera, disordered qi and blood, and brain vessel palsy. The disease location is the brain. Doctors of all dynasties consider that "the lesion is in the head, and governor meridian should be examined first”. Since governor meridian passes through Baihui acupoint and Dazhui is the confluence of hand and feet triple yang channel and governor meridian. Clinically, Baihui acupoint and Dazhui are often used to treat cerebral ischemia disease ${ }^{[2]}$. Thus, the above acupuncture points are chosen for electric acupuncture treatment to make all main and collateral channels through and balance yin and yang. Cell apoptosis is an autonomous and ordered cell death process due to synergetic adjustment of genes and relevant regulatory factors ${ }^{[3]}$. It is an important link which decides the development of cerebral ischemic injury. Hence, effective suppression of impaired nerve cell apoptosis and recovery of impaired brain tissue are important approaches to treat focal cerebral ischemia disease ${ }^{[4]}$. Bcl-xl is an important apoptosis suppression factor, while apoptosis finally depends on activation of Caspase-3. It is found in this study that Caspase-3 expression rises immediately after cerebral ischemia for $2 \mathrm{~h}$. As ischemia time increases, Caspase- 3 content gradually increases, and reaches the peak after cerebral ischemia for $3 \mathrm{~d}$. During the comparison in the same time bucket, Caspase-3 content of electric acupuncture is minimum. Acupuncturing Baihui point and 
Dazhui can effectively suppress neuronal apoptosis after focal cerebral ischemia and promote impaired brain tissue to recover.

The results show that after cerebral ischemia for $2 \mathrm{~h}-1 \mathrm{~d}$, Bcl-xl expression level of model group and electric acupuncture is high. This may be because body irritability improves Bcl-xl expression after cerebral ischemia so as to relieve the damage caused by cerebral ischemia and improve the ability to repair impaired nerve cell ${ }^{[5]}$. After cerebral ischemia for $3 \mathrm{~d}$, Bcl-xl content of model group starts to decline, and cell apoptosis reaches the peak. This may be because as cerebral ischemia time increases, the degree of impairment surpasses self-repairing capability of the body, and brain tissue impairment is exacerbated. After cerebral ischemia for 3d, Bcl-xl expression of electric acupuncture drops slightly, but it is still at a high level after cerebral ischemia for $7 \mathrm{~d}$, and higher than that of other groups in the same time bucket. Correspondingly, neuron damage degree is lower than that of other groups in the same time bucket. This may be because early intervention of acupuncturing can boost Bcl-xl activity of ischemia cortex, effectively adjust high-level Bcl-xl expression to maintain a long time, enhance suppression of time effect and dose-effect intensity of cell apoptosis after cerebral ischemia and give play to the treatment function of protection of impaired brain tissue. The research indicates that after focal cerebral ischemia, EPO starts phosphorylation of downstream factor STAT5 through mediating EPOR-JAK2-STAT5 signal transduction approach. The activated STAT5 can effectively up-regulate expression of Bcl-2, Bcl-xl and other inhibitors of apoptosis protein, benignly down-regulate expression of Bax, Caspase-3 and other pro-apoptotic protein, and effectively prevent cytochrome $\mathrm{C}$ from releasing from mitochondria so as to give play to the anti-apoptosis effect after cerebral ischemia ${ }^{[6-8]}$. The research result prompts that Acupuncturing Baihui point and Dazhui can effectively regulate expression of key target spots after cerebral ischemia and suppress neuronal apoptosis.

It is found in this research that, Caspase-3 content of AG490 group is higher than that of model group in the process of cerebral ischemic injury. After ischemia for $2 \mathrm{~h}$ and $1 \mathrm{~d}, \mathrm{Bcl}-\mathrm{xl}$ expression reaches the peak. The content of AG490+electric acupuncture group is only close to that of AG490 group, and significantly lower than that of model group and acupuncturing group in the same time bucket. AG490 is specificity inhibitor of JAK2 and suppresses JAK2 phosphorylation through competing for the receptor so as to block JAK2/STAT5 signal channel and restrain it to mediate transcription function of downstream factor ${ }^{[9]}$. The above result may be because after AG490 is injected, specificity suppresses JAK2 phosphorylation and blocks activation of JAK2/STAT5 signal channel so as to reduce Bcl-xl content. Meanwhile, Caspase-3 and Bcl-xl content of AG490+electric acupuncture group is similar to that of AG490 group. STAT5 is the interest protein of JAK2. When JAK2 is suppressed by specificity, Bcl-xl content does not increase after acupuncturing, and the regulation function for Bcl-xl and Caspase-3 is not significant. Thus, it is speculated that, up-regulation Bcl-xl expression level and down-regulation of Caspase-3 expression level to relieve protection function of cerebral ischemia injury may be achieved through starting JAK2/STAT5 signal transduction channel.

The research result indicates that focal cerebral ischemia facilitates suppression of neuronal apoptosis so as to relieve brain tissue impairment. Acupuncturing "Baihui" and "Dazhui" can effectively activate JAK2/STAT5 signal channel, stimulate self-protection mechanism of body and give play to cranial nerve protection function. However, since activation of JAK2 can mediate network of multiple intracellular transduction signal channels and participate in inflammatory response and cell apoptosis after cerebral ischemia. It is required to expound the mechanism of cerebral ischemic injury treatment by acupuncturing more comprehensively, analyze the overall function of regulating homeostasis survival signal cascade reaction of body through acupuncture and moxibustion. Further discussion will focus on regulation of key target point through acupuncturing and the effect on upstream and downstream factors. 


\section{Acknowledgments}

Fund project: Natural science fund project of Guangdong Province, No.: 2015A030310375; Scientific research topic of Chinese Traditional Medicine Bureau of Guangdong Province, No.: 20151232.

\section{References}

[1] Sun Shixiao, Liu Hongyu, Yang Chunzhuang et al. Influence of electric acupuncture on penumbra nerve cell apoptosis of rats with focal cerebral ischemia, and protein expression of relevant apoptosis genes Bcl-2, Bax and c-Fos , Acta Chinese Medicine and Pharmacology, 2011,39(1):65-68.

[2] Huang Kangbai, Xu Nenggui, Yi Wei et al., Influence of electric acupuncture on STAT5 expression in ischemia focus area of rats, 2011 collected papers of China Institute of Acupuncture and Moxibustion, 2011:253-262.

[3] LIU CM, LI RQ, SONG XL, et al. Effect of catgut implantation at acupoints on GABA_B and mGluR1 expressions in brain stem of rats with spasticity after stroke, $J$ Tradit Chin Med,2014, 34(5): 566-571.

[4] Li Li, Gu Zhengtao, Liu Zhifeng et al., Function of Caspase-3 in induction of human umbilical vein endothelial cell apoptosis by thermal shock, The Journal of Practical Medicine, 2014,30(6):871-874.

[5] KOSAN C, GINTER T, Heinzel T,et al. STAT5 acetylation:Mechanisms and consequences for immunological control and leukemogenesis, JAK-STAT, 2013,2(4):e26102-e26113.

[6] LETOURNEUR A, PETIT E, ROUSSEL S, et al. Brain ischemic injury in rodents : the protective effect of EPO , Methods Mol Biol, 2013(982):79-101.

[7] LIU GY, WANG T, WANG TG, et al. Effects of apoptosis-related proteins caspase-3, Bax and Bcl-2 on cerebral ischemia rats , Biomed Rep.2013,1(6):861-867.

[8] MILJUS N, HEIBECK S, JARRAR M,et al.Erythropoietin-mediated protection of insect brain neurons involves JAK and STAT but not PI3K transduction pathways, Neuroscience, 2014,258:218-227.

[9] Joung YH, Na YM, Yoo YB, et al. Combination of AG490, a Jak2 inhibitor, and methylsulfonylmethane synergistically suppresses bladder tumor growth via the Jak2/STAT3 pathway, Int J Oncol,2014, 44(3):883-895. 